\title{
Comparison of Vasopressin and Oxytocin Expressions in the Hypothalamo-Neurohypophysial System of Patients with Chronic Heart Failure
}

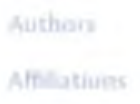

Key words

Q vasopressin

coxytocin

o hypothalamus

o chronic heart failure

\author{
E. v. Sivukhina , lu. E. Morozov , A. A. Dolzhikov , G. F. Jirikowski , V. Grinevich \\ Department of Anatomy II, Friedrich-Schiller University, Jena, Germany \\ Regional Medical Expert Bureau of Medical Legal Examination, Kaliningrad, Russia \\ Department of Pathology, Regional Hospital, Belgorod, Russia \\ Department of Molecular Neurobiology, Max-Planck-Institute for Medical Research, Heidelberg. Germany
}

tocin-immunoreactive neurons and their terminals in the posterior pituitary of 8 male patients (53.8 \pm 9.3 years) who had died from CHF and 9 male controls ( $54.6 \pm 11.8$ years). In line with previous clinical reports, our study on hypothalami of chronic heart failure patients revealed a significant increase in the relative profile density $(+29 \%)$ of vasopressin-positive neurons in the hypothalamic supraoptic nucleus. Consistently we found a significant increase in the relative optic density of vasopressin-immunoreactivity in the posterior pituitary ( $+33 \%)$ of these patients. In contrast, the similar analysis applied for oxytocin neurons revealed no statistically significant differences to controls. In conclusion, our study provides the morphological evidence for activation of vasopressin (but not oxytocin) expression and vasopressin transport to the posterior pituitary in patients with chronic heart failure.

\section{Introduction}

$\boldsymbol{T}$

Chronic heart failure (CHF) is considered as a principal problem in cardiology because of its extremely wide distribution all over the world and poor prognosis with high levels of lethality $[1,2]$. CHF is a highly complex clinical syndrome, characterized mainly by progressive failure of heart pump function and body fluid accumulation. The nonapeptide neurohormone vasopressin (VP), one of the main physiological regulators of water and electrolyte balance, is synthesized mainly in magnocellular hypothalamic nuclei, supraoptic (SON) and paraventricular (PVN) nuclei, and released into peripheral blood circuitry from the posterior pituitary lobe in response to increased plasma osmolality or hypovolemia [3]. Elevated levels of VP in the blood of CHF patients are well documented and are related to the severity and the poor prognosis of the disease [4-6]. Although VP is considered as an important neuroendocrine pathogenetic factor of CHF [7 and references cited therein], its expression in the hypothalamic nuclei and/or its transport to the posterior pituitary in CHF patients has not yet been investigated.

Despite the extensive clinical studies focused on the role of VP in the pathogenesis of CHF, the impact of another member of the hypothalamic nonapeptide family - oxytocin (OT) - to this disease has not been evaluated. Although it is well documented that OT contributes to the regulation of the water-electrolyte balance [8-11] and cardiovascular responses [12-15], analysis of OT plasma levels as well as OT expression in the hypothalamus of CHF patients has not been performed so far. To fill in this gap, we focused here on the comparative immunohistochemical and morphometric analysis of VP and OT expression in the hypothalamus and the content of the both nonapeptides in the posterior pituitary using postmortem samples obtained from subjects who died from CHF. 


\section{Materials and Methods}

$\mathbf{7}$

Tissue collection

Brains from patients were obtained according to the Russian law for autopsy from the Regional Medical Expert Bureau of Medical Legal Examination (Kaliningrad, Russia) and from an Emergency Hospital (Kursk, Russia). Hypothalami and pituitaries of eight male patients (mean age $53.8 \pm 9.3$ years) who suffered from $\mathrm{CHF}$ and died due to acute heart or respiratory failure were compared to samples of brains from nine male individuals (mean age $54.6 \pm 11.8$ years) without known primary neurological or psychiatric conditions and endocrinological pathology who died from non-CHF related causes: acute brain stroke (3), diseases of the respiratory system (2), acute surgical pathology (2), asphyxia (1), and traumatic injury (1). Postmortem time was $15.8 \pm 6.4 \mathrm{~h}$ in controls and $22.5 \pm 11.7 \mathrm{~h}$ in CHF group [16]. In all cases CHF was verified by autopsy by several pathological criteria: hypertrophy and lipomatosis of the myocardium with dilated heart chambers; local hypertrophy, dystrophy, or atrophy of cardiomyocytes with their lysis and subsequent cardiosclerosis; injuries of blood capillaries with an increase of the wall thickness; and activation of fibroblasts. Tissue preparation was done as described [17]. The study was approved by the Local Ethics Committee of the Friedrich-Schiller University. Jena, Germany (Protocol 2387-09/08).

\section{Imumunohistochemistry}

Consecutive 6-8 $\mu \mathrm{m}$ paraffin sections of pituitaries and hypothalami containing PVN and SON were stained with antibodies against VP or OT as described [16,17]. Briefly, overnight incubation with one of the primary rabbit polyclonal antibodies - antiVP or anti-OT (Chemicon International, CA, USA: 1:1000) - in working solution (WS; $1 \%$ normal goat serum, $0.05 \%$ Triton in PBS, $\mathrm{pH}$ 7.4) was performed in a humid chamber at $4{ }^{\circ} \mathrm{C}$. Incubation in WS with biotinylated goat anti-rabbit IgG (Vector Elite kit, Vector Laboratories, Inc., Burlingame, CA, USA: 1:200) for $2 \mathrm{~h}$ at room temperature was followed by application of $A B C$ complex (Vector Laboratories, CA) in PBS ( 2 h, r.t.). The reaction was visualized with freshly prepared diaminobenzidine (DAB, Fast kit, Sigma) and $\mathrm{H}_{2} \mathrm{O}_{2}$ in PBS for $10 \mathrm{~min}$. After rinsing with distilled water, sections were dehydrated through ascending concentrations of ethanol, cleared in xylene, and mounted with embedding medium (Entellan, Merck, Darmstadt, Germany). For control incubations we used normal goat serum, diluted 1:1000 in PBS-Triton $\mathrm{X}$ instead of the primary antibodies.

\section{Quantitative analysis of VP. and OT-positive neurons in} the hypothalamic nuclei

Counting of VP- and OT-positive neurons was performed on an Axioskop microscope (Zeiss, Oberkochen, Germany) equipped with a motorized stage and Neurolucida software-controlled computer system (MicroBrightField Europe, Magdeburg, Germany) as described [16]. Briefly, the contours of the fields of hypothalamic paraventricular (PVN) or supraoptic (SON) nuclei containing VP- or OT-positive neurons were outlined manually and all immunopositive neurons were labeled by symbol and counted. Finally, cell profile densities (numbers of cell profiles per unit area) of VP- or OT-positive cells were calculated. Additionally, an average nearest neighbor distance between immunoreactive (IR) neurons was estimated using Neuroexplorer software (MicroBrightField Europe, Magdeburg, Germany), All counts were performed blindly in coded preparations by the same investigator.

\section{Quantification of VP. or OT-IR in neurons and in the posterior pituitary}

Areas and spectra of optic density (OD) of DAB-IR products in neurons and nerve terminals were defined in immunocytochemically stained sections as described [17] using the "ImageTool" (University of Texas Health Science Center, USA) and "ImageJ" (NIH) software packages, Relative OD of VP- or OT-IR in the posterior pituitary was estimated as correlations of measured area (in pixels) and average density of the grey level (in arbitrary units, a.u.). Areas of the cell somata and nuclei of neurons were measured in the sections stained for VP or OT. After that, absolute and relative (in \%) areas of IR products were calculated.

\section{Photographical documentation}

Immunostained VP and OT sections were examined with an "Olympus BX50" microscope. An "Olympus DP10" digital camera with "DP Soft 3.0" software was used for microphotography. Digital images were adjusted for linear contrast and brightness in Adobe Photoshop (Adobe Systems, version 8.0.1).

\section{Statistical analysis}

The nonparametric Mann-Whitney U-test was applied to evaluate the morphological data. All results are presented as a group mean values with standard deviation (SD). The accepted level of significance was $5 \%$.

\section{Results \\ $\nabla$}

Effects of chronic heart failure on the magnocellular VP system

Immunostaining for VP revealed a similar cellular distribution within the PVN and SON in both control and CHF groups. In control patients VP-positive neurons were predominantly found as weak- to moderate-labeled cells within the magnocellular hypothalamic nuclei ( $\triangle$ Fig. 1A, B). Analysis of these nuclei in the CHF patients revealed significantly higher intensity of VP-IR as compared with the controls ( 0 Fig. 1D, E). Moreover, in addition to the cell bodies, numerous cell processes were also heavily stained especially in the SON of CHF patients (o Fig. 1E). Next, we quantified the expression of VP in the magnocellular hypothalamic nuclei. As indicated in Fig. 1G, increased density of VP-positive cell profiles was observed in the both PVN and SON of CHF patients as compared to the controls. This increase was statistically significant only in the SON $(+29 \%, p<0.05)$. The content of VP-IR, estimated as relative area of IR product in neurons, was slightly, but also statistically significantly $(p<0.05)$ increased in the SON of CHF patients $(+6.97 \%)$. Additionally, the spatial distribution of VP-IR neurons was evaluated by a nearest neighbor distance analysis, which revealed a reduction in the distance between VP-positive cell profiles in the magnocellular hypothalamic nuclei in CHF patients ( $O$ Fig. $1 \mathrm{H}$ ) and correlated well with the cell profile density (o Fig. 1G).

In the posterior pituitaries of controls relatively equal and moderate distribution of VP-IR was observed ( $\circ$ Fig. 1C), In contrast, in CHF patients we found irregular distribution and enhanced intensity of VP-IR signal ( $\circ$ Fig. 1F). Semiquantitative analysis of relative OD of VP-IR in the posterior pituitary revealed signifi- 

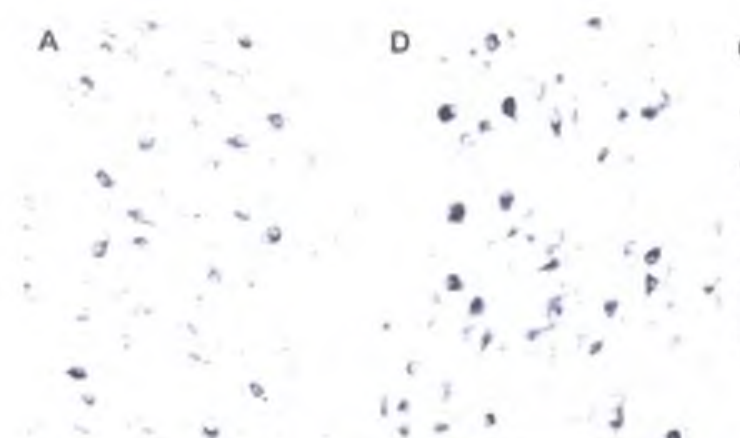

G
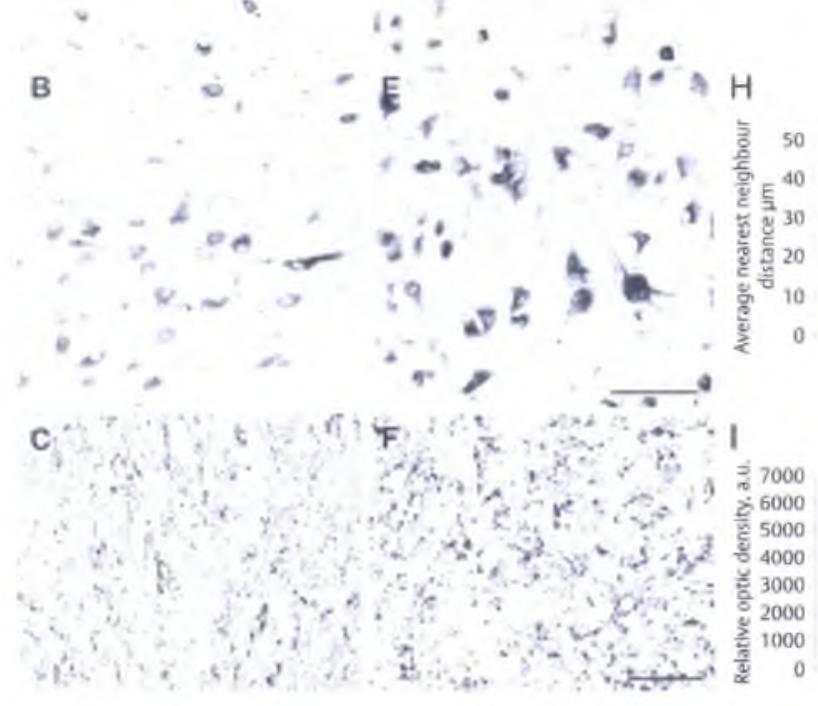

cant increase in the CHF group as compared with the controls $(+33 \%, p<0.05$; ○ Fig. 11).

\section{Effects of chronic heart failure on the magnocellular or system}

The anatomical distribution of OT-positive cells within the PVN (O Fig, 2A, D) and SON ( $\circ$ Fig. 2B, E) was similar in both studied groups. The population of OT-IR neurons in the PVN was relatively equal in size and OT-IR cells were scattered over a large area of the nucleus in the hypothalami of controls (o Fig. 2A) and CHF patients ( $\circ$ Fig. 2D). However, the intensity of OTstaining of cell bodies and their processes in the control group varied from weak to very strong ( 0 Fig. 2A), while in the PVN of CHF patients moderately stained cells prevailed (o Fig. 2D). In the SON the population of OT-positive cells was much smaller in comparison with the PVN in both control (O Fig. 2B) and CHF groups (o Fig. 2E). OT-IR appeared as clear homogeneous cytoplasmic staining pattern. Semiquantitative analysis of profile density of OT-positive neurons revealed no statistically significant difference between controls and CHF patients either in the PVN or in the SON, as shown in o Fig. 2G. A nearest neighbor distance analysis indicated that the distance between OT-IR neurons was slightly, but not significantly, increased in the magnocellular hypothalamic nuclei of CHF patients ( 0 Fig. $2 \mathrm{H}$ ). Analysis of OT content in the magnocellular neurons, estimated as relative area of IR product, within the PVN and SON also revealed no statistically significant differences between control and CHF groups (data not shown).

In the posterior pituitaries of controls (o Fig. 2C) and CHF patients (o Fig. 2F) we observed relatively equal and moderate
Fig. 1 Eflect of $\mathrm{CHF}$ on magnocellular VP. IR neurons and their terminals in the posterior pituitary. The PVN (A) and SON (B) of a 42-year old control and a 65 -year old CHF patient (D. E). Note numerous intensively stained VP-positive neurons and their processes especially in the SON of patient suffered from CHF (E) compared to control (B), VP-IR in the posterior pitultary of a 65 -year old CHF patient (F) is increased in comparison to a 70 year old control (C). Immunostaining with anti-VP antibodies, developed by DAB. Scale bars $=100 \mu \mathrm{m}$ (A, B, D, E) and $50 \mu \mathrm{m}(\mathbf{C}, \mathbf{F}), \mathbf{G}$. Relative cell profile density of VP-positive neurons is significantly higher in CHF group than in controls. H: The average nearest neighbor distance between VP. positive cells is smaller in CHF patlents. I: Significant increase of OD for VP-IR occurred in the posterior pituitary of CHF patients. Group mean values of the parameters are calculated from individual mean values. " $p<0,05$. distribution of OT-IR with no difference in the relative OD (o Fig. 21),

\section{Discussion}

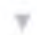

In the present study we report the profound activation of VP expression in the hypothalamo-neurohypophyseal system in patients afflicted with CHF. Results of our morphological analysis indicate an increase in the density of magnocellular VP-positive neurons within the PVN and SON. This increase has reached significance in the SON (but not in the PVN), known as a major source of magnocellular VP in the human hypothalamus [18]. Present analysis of the hypothalamo-neurohypophyseal system in CHF subjects showed not only the elevation of VP expression in magnocellular neurons (more profoundly in the SON), but also an increase of VP transport to the posterior pituitary. These findings are consistent with numerous clinical studies, which demonstrated a significant elevation of VP levels in patients with CHF and its deleterious effects on left ventricular remodeling, vasoconstriction, and water retention [4-6, 19].

It is known that VP regulates vascular tone and free-water reabsorption through the VP receptor subtype V1 and V2, respectively $[20,21]$. From such a point of view, the interfering with VP-mediated signaling may be beneficial pharmacologically. Novel preclinical and clinical trial results indicate that VP receptors antagonism (selective V2 or combined V2/V1 blockers) is a highly promising and rational approach for the treatment of major symptoms of CHF [for reviews, see 22,23]. Recently performed comprehensive study in the framework of the Efficacy of 


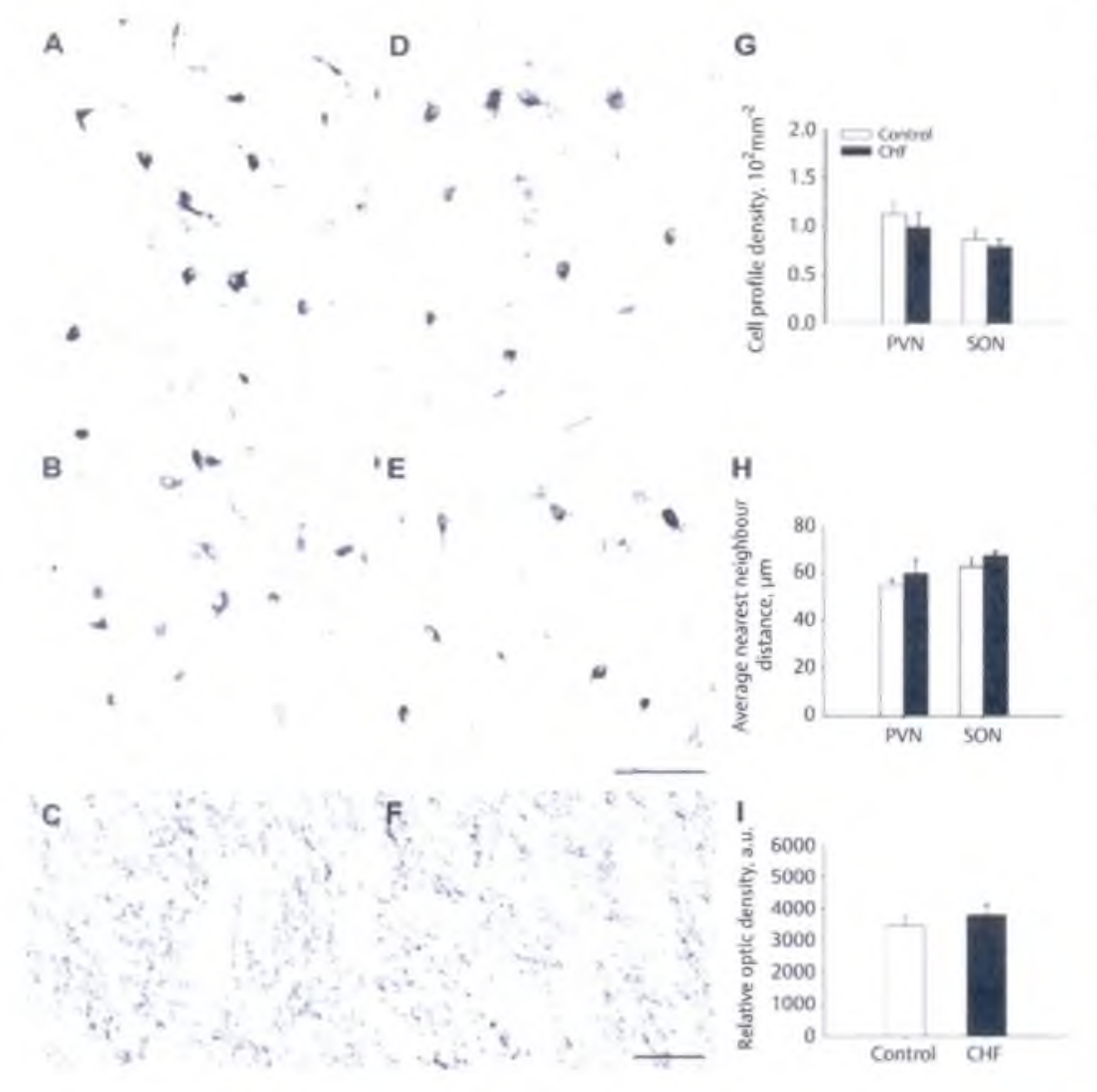

Fig. 2 Effect of CHF on magnocellular OTIR neurons and their terminals in the posterior pituitary. Relatively low and equal number of OT. positive neurons in the PVN of a 64 year old control (A) and a 65-year old CHF patient (D), Moderate and weak OT expression in the SON of a 67 -year old control (B) and a 65 year old CHF patient (E). OTAR in the posterior pituitary of a 70-year old control (C) and a 65-year old CHF patient (F). Immunostaining with anti-OT antibodies, developed by DAB. Scale bars $=100 \mu \mathrm{m}$ (A, B, D, E) and $50 \mu \mathrm{m}$ (C, F). G: No statistically significant differences in the relative cell profile density of OT-positive neurons in CHF group compared to controls. $\mathbf{H}$ : The average nearest neighbor distance between OT-positive cells is slightly higher in the SON of CHF patients. I: Relative OD of OT-IR in the posterior pituitary of CHF patients is statistically not different compared to controls. Group mean values of the parameters are calculated from individual mean values.

Vasopressin Antagonism in Heart Failure Outcome Study With Tolvaptan (EVEREST) program showed high efficiency of the selective VP V2 receptor antagonist application for amelioration of CHF symptoms without serious adverse events [24]. The third subtype of VP receptors, V3, is expressed mainly in the pituitary. Its activation by VP leads to the increase of synthesis of adrenocorticotropin hormone (ACTH) $[25,26]$. Although the origin of VP acting on pituitary ACTH synthesis is mostly the parvocellular portion of the PVN, it can not be excluded that magnocellular VP neurons also impact on stimulation of ACTH synthesis [27]. Therefore the activation of VP expression in magnocellular neurons may contribute to an increase of plasma ACTH levels [28] as well as to elevation of ACTH-IR in the anterior pituitary of CHF patients [16].

In contrast to VP, we did not observe significant changes in any analyzed parameters applied for OT system in CHF patients in the present study. Despite the growing body of literature on the contribution of OT in cardiovascular [12-15], osmotic [8-11]. and stress [29-31] responses, we failed to detect at the anatomical level any impairment of OT neurons and their axonal terminals in the posterior pituitary of CHF patients. However, our morphological data do not exclude alterations in the tremendously complex actions of oT [32 and references cited therein] within the brain of CHF patients. In addition, further measurement of OT plasma levels in patients of different functional classes of $\mathrm{CHF}$ will provide valuable information.

In conclusion we have demonstrated that while expression of VP in magnocellular neurons and its transport to the posterior pituitary were significantly increased in CHF patients, OT-IR neurons remained morphologically unaffected by this ailment.

\section{Acknowledgements \\ $\checkmark$}

The authors thank Prof. Dr. Otto W. Witte for use of the microscopic facility in the Department of Neurology, Friedrich-Schiller University Jena and Roswitha Kasch for technical assistance. E.S. was a recipient of DAAD short-term fellowship.

\section{References}

1 Kannel WB, Belanger AJ. Epidemiology of Heart Failure. Am Heart J 1991; 121: 951-957

2 McMurray, Pfeffer MA. Heart failure Lancet 2005; 365: 1877-1889

3 Robertson GL. Physiology of ADH secretion. Kidney Int 1987; 21 (Suppl): \$20-\$26

4 Dudoev VA, Gorin V, Borodkin VV, Diukov IV, Nechaeva NI. Vasopressin content of the blood in ischemic heart disease patients and its interrelation with other hormones. Kardiologiia 1986; 26: 98-101

5 Kjaer A, Hesse B. Heart failure and neuroendicrine activation: diagnostic, prognostic and therapeutic perspectives. Clin Physiol 2001; 21: $661-672$

6 Nakamura T, Funayama H. Yoshimura A, Tsuruya $Y$, Saito M, Kawakami M. Ishikawa SE. Possible vascular role of increased plasma arginine vasopressin in congestive heart failure. Int J Cardiol 2006: 106 191-195

7 Lejemtel TH, Serrano C. Vasopressin dysregulation: hyponatremia, fluid retention and congestive heart failure. Int J Cardiol 2007; 120: 1-9

8 Ventura RR, Gomes DA, Reis WL, Elias $L$, Castro M, Valença MM, Carnio EC. Rettori V. McCann SM, Antunes-Rodrigues J. Nitrergic modulation of vasopressin. oxytocin and atrial natriuretic peptide secretion in response to sodium intake and hypertonic blood volume expansion. Braz J Med Biol Res 2002; 35: 1101-1109

9 Lauand F, Ruginsk SG, Rodrigues HL, Reis WL, de Castro M, Elias $U_{\text {, }}$ Antunes-Rodrigues J. Glucocorticoid modulation of atrial natriuretic peptide, oxytocin, vasopressin and Fos expression in response to osmotic, angiotensinergic and cholinergic stimulation. Neuroscience 2007: 147: 247-257 
10 Chou CL, DiGiovanni SR, Mejia R, Nielsen S, Knepper MA. Oxytocin as an antidiuretic hormone. Am J Physiol 1995; 269 (1 Pt 2): F70-F77. F78-F85

11 Hoanwinckel MA. Elias LK, Favaretto AL, Gutkowska J. McCann SM. Antunes-Rodrigues $J$. Oxytocin mediates atrial natriuretic peptide release and natriuresis after volume expansion in the rat. Proc Nat Acad Sci USA 1995: 92: 7902-7906

12 Buijs RM. Swaab DF. Immuno-electron microscopical demonstration of vasopressin and oxytocin synapses in the limbic system. Cell Tissue Res 1979; 204: 355-365

13 Sawchenko PE, Swanson LW. Immunohistochemical identification of neurons in the paraventricular nucleus of hypothalamus that project to the medulla or to the spinal cord in the rat. J Comp Neurol 1982; 205: 260-272

14 Maier T, Dai WJ. Csikós T, Jirikowski GF, Unger T, Culman J. Oxytocin Pathways Mediate the Cardiovascular and Behavioral Responses to Substance $P$ in the Rat Brain. Hypertension 1998; 31: 480-486

15 Wsol A, Cudnoch-Jedrzejewska A.Szczepanska-Sadowska E. Kowalewski 5. Dobruch J. Central oxytocin modulation of acute stress-induced cardiovascular responses after myocardial infarction in the rat. Stress 2009 Feb; 9: 1 [Epub ahead of print]

16 Sivukhina EV, Poskrebysheva AS, Smurova luV, Dolzhikov AA, Morozov IuE, Jirikowski GF, Grinevich V. Altered hypothalamic-pituitary-adrenal axis activity in patients with chronic heart failure. Horm Metab Res 2009: 41: in press

17 Sivukhina EV. Dolzhikov AA, Morozov IuE, Jirikowski GF, Grinevich V. Effects of chronic alcoholic disease on magnocellular and parvocellular hypothalamic neurons in men. Horm Metab Res 2006: 38: 382-390

18 Swaab DF. Neurobiology and neuropathology of the human hypothalamus. In: Bloom FE, Bjorklund A. Hökfelt T, eds Handbook of Chemical Neuroanatomy Vol. 13. Amsterdam: Elsevier; 1997; 39-138

19 Goldsmith SR. Vasopressin receptor antagonists: mechanisms of action and potential effects in heart failure. Cleve Clin J Med 2006; 73 (Suppl 2): $\$ 20-\$ 23$

20 Chatterjee $K$. Neurohormonal activation in congestive heart failure and the role of vasopressin. Am J Cardiol 2005; 95: 8B-13B

21 Oh MS. Management of hyponatremia and clinical use of vasopressin antagonists. Am J Med Sci 2007: 333: 101-105
22 Sanghi $P$, Uretsky BF, Schwarz ER. Vasopressin antagonism: a future treatment option in heart failure. Eur Heart J 2005; 26: 538-543

23 Rai A. Whaley-Connell A. McFarlane S, Sowers JR. Hyponatremia, Arginine Vasopressin dysregulation, and Vasopressin receptor antagonism. Am J Nephrol 2006: 26: 579-589

24 Gheorghiade M, Konstam MA, Burnetr Jr. JC, Grinfeld L, Maggioni AP. Swedberg K, Udelson JE, Zannad F, Cook T, Ouyang J. Zimmer C, Orlandi C. Short-term clinical effects of tolvaptan, an oral vasopressin antagonist, in patients hospitalized for heart failure: the EVEREST Clinical Status Trials. JAMA 2007; 297: 1332-1343

25 Burrell LM. Risvanis J. Johnston Cl. Naitoh M, Baldinq LC. Vasopressin receptor antagonism: a therapeutic option in heart failure and hypertension. Exp Physiol 2000; 85 (Suppl): 259S-265S

26 Ramos AT, Troncone LR, Tufik S. Suppression of Adrenocorticotrophic Hormone Secretion by Simultaneous Antagonism of Vasopressin Ib and CRH-1 Receptors on Three Different Stress Models. Neuroendocrinology 2006: 84: 309-316

27 Holmes MC, Antoni FA, Aguilera G, Catt KJ. Magnocellular axons in passage through the median eminence release vasopressin. Nature 1986; 319: 326-329

28 Kasarkina LV. Pivovarov VN, Markova EV, Salem S. Rossel's AN. Blood hormones in chronic ischemic heart disease and acute myocardial infarct. Kardiologiia 1979; 19: 93-98

29 Jezova D, Skultetyova I, Tokarev DI, Bakos P, Vigas M. Vasopressin and oxytocin in stress. Ann NY Acad Sci 1995: 771: 192-203

30 Engelmann $M$. Landgraf $R$. Wotjak CT. The hypothalamic-neurohypophyseal system regulates the hypothalamic-pituitary adrenal axis under stress: an old concept revisited. Front Neuroendocrinol 2004: 25: 132-149

31 Laguna-Abreu MT, Koenigkam-Santos $M$, Colleta AM, Elias PC, Moreira $A C$. Antunes-Rodrigues J. Elias $L_{\text {. }}$ Castro $M$. Time course of vasopressin and oxytocin secretion after stress in adrenalectomized rats. Horm Metab Res 2005; 37: 84-88

32 Landgraf $R$. Neumann ID. Vasopressin and oxytocin release within the brain: a dynamic concept of multiple and variable modes of neuropeptide communication. Front Neuroendocrinol 2004; 25: 150-176 